\title{
Application of Lie Symmetry Analysis and Simplest Equation Method for Finding Exact Solutions of Boussinesq Equations
}

\author{
Hossein Jafari, ${ }^{1,2}$ Nematollah Kadkhoda, ${ }^{1}$ and Chaudry Massod Khalique ${ }^{2}$ \\ ${ }^{1}$ Department of Mathematics, University of Mazandaran, Babolsar 47416-95447, Iran \\ ${ }^{2}$ International Institute for Symmetry Analysis and Mathematical Modeling, Department of Mathematical Sciences, \\ North-West University, Mafikeng Campus, Private Bag X 2046, Mmabatho 2735, South Africa
}

Correspondence should be addressed to Hossein Jafari; jafari@umz.ac.ir

Received 18 May 2013; Revised 28 June 2013; Accepted 8 July 2013

Academic Editor: Fazal M. Mahomed

Copyright (c) 2013 Hossein Jafari et al. This is an open access article distributed under the Creative Commons Attribution License, which permits unrestricted use, distribution, and reproduction in any medium, provided the original work is properly cited.

The Lie symmetry approach with simplest equation method is used to construct exact solutions of the bad Boussinesq and good Boussinesq equations. As the simplest equation, we have used the equation of Riccati.

\section{Introduction}

Nonlinear wave phenomena, which are modelled by nonlinear partial differential equations (NLPDEs), appear in various scientific and engineering fields, such as fluid mechanics, plasma physics, optimal fiber, biology, solid state physics, chemical physics, geometry, and oceanology [1-15]. Much effort has been made on the construction of exact solutions of NLPDEs. These nonlinear equations have been studied by using various analytical methods, such as tanh-function method, extended tanh-function method [1-3], sine-cosine method [4, 5], $\left(G^{\prime} / G\right)$-expansion method [6], and so on. In this paper, we study the Boussinesq equations [7]:

$$
\begin{gathered}
u_{t t}-u_{x x}-3\left(u^{2}\right)_{x x}-u_{x x x x}=0, \text { bad equation, } \\
u_{t t}-u_{x x}-3\left(u^{2}\right)_{x x}+u_{x x x x}=0, \text { good equation, }
\end{gathered}
$$

which are named after the French scientist Joseph Boussinesq (1842-1929). These equations were modelled in the 1870 s and they describe the propagation of long waves on the surface of water with a small amplitude. The Boussinesq equations have been solved using several methods [8-11]. In this paper, we use the Lie symmetry method along with the simplest equation method to obtain exact solutions of the Boussinesq equations (1)-(2). The simplest equation method was developed by Kudryashov [12] on the basis of a procedure analogous to the first step of the test for the Painlevé property. The outline of this paper is as follows.

In Section 2, we discuss the methodology of the simplest equation method when the simplest equation is the equation of Riccati. In Section 3, we discuss the symmetry analysis, and in Section 4, we obtain exact solutions of the mentioned Boussinesq equations. Concluding remarks are summarized in Section 5.

\section{Analysis of the Simplest Equation Method}

We consider a partial differential equation and assume that by means of an appropriate transformation this partial differential equation is transformed to a nonlinear ordinary differential equation in the form

$$
P\left(F, F^{\prime}, F^{\prime \prime}, F^{\prime \prime \prime}, \ldots\right)=0 .
$$

Exact solution of this equation can be constructed as finite series

$$
F(\xi)=\sum_{i=0}^{n} A_{i}(G(\xi))^{i},
$$

where $G(\xi)$ is a solution of some ordinary differential equation referred to as the simplest equation. The simplest equation has two properties:

(1) the order of simplest equation should be less than the order of (3); 
(2) we should know the general solution of the simplest equation or at least exact analytical particular solution(s) of the simplest equation.

In this paper, we use the equation of Riccati as the simplest equation. This equation is a well-known nonlinear ordinary differential equation which has exact solutions in terms of elementary functions. In this paper, for the Riccati equation

$$
G^{\prime}(\xi)=c G(\xi)+d G(\xi)^{2},
$$

where $c$ and $d$ are nonzero constants, we use the solution

$$
\begin{aligned}
& G(\xi)=\frac{c \exp \left[c\left(\xi+\xi_{0}\right)\right]}{1-d \exp \left[c\left(\xi+\xi_{0}\right)\right]}, \quad \text { for } d<0, c>0, \\
& G(\xi)=-\frac{c \exp \left[c\left(\xi+\xi_{0}\right)\right]}{1+d \exp \left[c\left(\xi+\xi_{0}\right)\right]}, \quad \text { for } d>0, c<0 .
\end{aligned}
$$

Here, $\xi_{0}$ is a constant of integration. Now, $F(\xi)$ can be determined explicitly by using the following three steps.

Step 1. By considering the homogeneous balance between the highest nonlinear terms and the highest order derivatives of $F(\xi)$ in (3), the positive integer $n$ in (4) is determined.

Step 2. By substituting (4) into (3), making use of (5), and collecting all terms with the same powers of $G$ together, the left-hand side of (3) is converted into a polynomial. After setting each coefficient of this polynomial to zero, we obtain a set of algebraic equations in terms of $A_{i}(i=0,1,2, \ldots, n)$.

Step 3. Solving the system of algebraic equations and then substituting the results and the general solutions (6) or (7) into (4) gives solutions of (3).

\section{Lie Symmetry Analysis}

To apply the classical method of symmetry analysis [16, 17], we consider the one-parameter Lie group of infinitesimal transformations in $x, t, u$ given by

$$
\begin{aligned}
& x^{*}=x+\epsilon \xi^{1}(x, t, u)+O\left(\epsilon^{2}\right), \\
& t^{*}=t+\epsilon \xi^{2}(x, t, u)+O\left(\epsilon^{2}\right), \\
& u^{*}=u+\epsilon \phi^{1}(x, t, u)+O\left(\epsilon^{2}\right),
\end{aligned}
$$

where $\epsilon$ is the group parameter. The related Lie algebra is generated by the vector field

$$
X=\xi^{1}(x, t, u) \frac{\partial}{\partial x}+\xi^{2}(x, t, u) \frac{\partial}{\partial t}+\phi^{1}(x, t, u) \frac{\partial}{\partial u} .
$$

Applying the fourth prolongation of the vector field (9), $X^{[4]}$, to (1), we have

$$
\left.X^{[4]}\left(u_{t t}-u_{x x}-3\left(u^{2}\right)_{x x}-u_{x x x x}\right)\right|_{(1)}=0 .
$$

Expanding the above equation, we obtain the following overdetermined system of linear partial differential equations:

$$
\begin{gathered}
\xi_{u}^{1}=0, \\
\xi_{u}^{2}=0, \\
\phi_{u u}^{1}=0 \\
\xi_{t}^{1}=0 \\
\xi_{x}^{2}=0, \\
6 u \xi_{x x}^{1}-12 \phi_{x}^{1}+\xi_{x x}^{1}-12 u \phi_{x u}^{1}-2 \phi_{x u}^{1}+\xi_{x x x x}^{1}-4 \phi_{x x x u}^{1}=0, \\
\phi_{t t}^{1}-6 u \phi_{x x}^{1}-\phi_{x x}^{1}-\phi_{x x x x}^{1}=0, \\
3 \phi^{1}-6 u \xi_{x}^{1}-\xi_{x}^{1}+6 u \xi_{t}^{2}+\xi_{t}^{2}-2 \xi_{x x x}^{1}+3 \phi_{x x u}^{1}=0, \\
2 \phi_{x u}^{1}-3 \xi_{x x}^{1}=0 \\
2 \xi_{t}^{2}-2 \xi_{x}^{1}+\phi_{u}^{1}=0 \\
\xi_{t}^{2}-2 \xi_{x}^{1}=0 \\
2 \phi_{t u}^{1}-\xi_{t t}^{2}=0 .
\end{gathered}
$$

Solving the above system of equations, we obtain the following three Lie point symmetries of (1):

$$
\begin{gathered}
X_{1}=\frac{\partial}{\partial t}, \quad X_{2}=\frac{\partial}{\partial x} \\
X_{3}=(1+6 u) \frac{\partial}{\partial u}-6 t \frac{\partial}{\partial t}-3 x \frac{\partial}{\partial x} .
\end{gathered}
$$

We now use the two translation symmetries $X_{1}$ and $X_{2}$ and consider $X=X_{1}+v X_{2}$. This symmetry $X$ yields the two invariants

$$
\xi=x-v t, \quad u=F,
$$

which gives a group invariant solution $u=F(\xi)$, and consequently using these invariants (1), is transformed into the fourth-order nonlinear ordinary differential equation

$$
\left(\beta^{2}-1\right) F^{\prime \prime}-3\left(F^{2}\right)^{\prime \prime}-F^{(4)}=0 .
$$

Likewise, (2) is transformed to

$$
\left(\beta^{2}-1\right) F^{\prime \prime}-3\left(F^{2}\right)^{\prime \prime}+F^{(4)}=0 .
$$

\section{Exact Solutions of the Boussinesq Equations}

We now use the simplest equation method to obtain exact solutions. Let us consider the solutions of (14) and (15) in the form

$$
F(\xi)=\sum_{i=0}^{n} A_{i}(G(\xi))^{i}
$$


where $G(\xi)$ satisfies the Riccati equation (5), $n$ is a positive integer that can be determined by a balancing procedure, and $A_{0}, A_{1}, A_{2}, \ldots, A_{n}$ are parameters to be determined.

In this case, the balancing procedure yields $n=2$, and so the solutions of (14) and (15) are of the form

$$
F(\xi)=\sum_{i=0}^{2} A_{i}(G(\xi))^{i}
$$

4.1. Solutions of (1) Using Simplest Equation Method. Substituting (17) into (14), making use of the Riccati equation (5) and then equating all coefficients of the functions $G^{i}$ to zero, we obtain an algebraic system of equations in terms of $A_{0}$, $A_{1}$, and $A_{2}$. Solving the algebraic system of equations, with the aid of mathematica, we obtain the following values of $A_{0}$, $A_{1}, A_{2}$ :

$$
\begin{gathered}
A_{0}=\left(\frac{1}{6}\right)\left(v^{2}-c^{2}-1\right), \\
A_{1}=-2 c d, \\
A_{2}=-2 d^{2} .
\end{gathered}
$$

Therefore, for the case $d<0, c>0$, the solution of (1) is given by

$$
\begin{aligned}
u_{1}(x, t)= & A_{0}+A_{1} \frac{c \exp \left[c\left(\xi+\xi_{0}\right)\right]}{1-d \exp \left[c\left(\xi+\xi_{0}\right)\right]} \\
& +A_{2}\left(\frac{c \exp \left[c\left(\xi+\xi_{0}\right)\right]}{1-d \exp \left[c\left(\xi+\xi_{0}\right)\right]}\right)^{2},
\end{aligned}
$$

and when $d>0, c<0$, the solution of (1) is given by

$$
\begin{aligned}
u_{2}(x, t)= & A_{0}-A_{1} \frac{c \exp \left[c\left(\xi+\xi_{0}\right)\right]}{1+d \exp \left[c\left(\xi+\xi_{0}\right)\right]} \\
& +A_{2}\left(\frac{c \exp \left[c\left(\xi+\xi_{0}\right)\right]}{1+d \exp \left[c\left(\xi+\xi_{0}\right)\right]}\right)^{2},
\end{aligned}
$$

where $\xi=x-v t$.

4.2. Solutions of (2) Using Simplest Equation Method. Following the same procedure as above, for (15), we obtain the following values of $A_{0}, A_{1}, A_{2}$ :

$$
\begin{gathered}
A_{0}=\left(\frac{1}{6}\right)\left(c^{2}+v^{2}-1\right), \\
A_{1}=2 c d, \\
A_{2}=2 d^{2},
\end{gathered}
$$

and so for the case $d<0, c\rangle 0$, the solution of (2) is given by

$$
\begin{aligned}
u_{1}(x, t)= & A_{0}+A_{1} \frac{c \exp \left[c\left(\xi+\xi_{0}\right)\right]}{1-d \exp \left[c\left(\xi+\xi_{0}\right)\right]} \\
& +A_{2}\left(\frac{c \exp \left[c\left(\xi+\xi_{0}\right)\right]}{1-d \exp \left[c\left(\xi+\xi_{0}\right)\right]}\right)^{2}
\end{aligned}
$$

and for $d>0, c<0$ the solution of (2) is given by

$$
\begin{aligned}
u_{2}(x, t)= & A_{0}-A_{1} \frac{c \exp \left[c\left(\xi+\xi_{0}\right)\right]}{1+d \exp \left[c\left(\xi+\xi_{0}\right)\right]} \\
& +A_{2}\left(\frac{c \exp \left[c\left(\xi+\xi_{0}\right)\right]}{1+d \exp \left[c\left(\xi+\xi_{0}\right)\right]}\right)^{2},
\end{aligned}
$$

where $\xi=x-v t$.

\section{Concluding Remarks}

In this paper, Lie symmetry method along with the simplest equation method has been successfully used to obtain exact solutions of the bad and good Boussinesq equations. As the simplest equation, we have used the equation of Riccati. We have also verified that the solutions we have found are indeed solutions to the original equations.

\section{References}

[1] E. Fan, "Extended tanh-function method and its applications to nonlinear equations," Physics Letters A, vol. 277, no. 4-5, pp. 212-218, 2000.

[2] A.-M. Wazwaz, "The tanh-coth method for solitons and kink solutions for nonlinear parabolic equations," Applied Mathematics and Computation, vol. 188, no. 2, pp. 1467-1475, 2007.

[3] A.-M. Wazwaz, "The tanh method: solitons and periodic solutions for the Dodd-Bullough-Mikhailov and the TzitzeicaDodd-Bullough equations," Chaos, Solitons and Fractals, vol. 25, no. 1, pp. 55-63, 2005.

[4] A.-M. Wazwaz, "A sine-cosine method for handling nonlinear wave equations," Mathematical and Computer Modelling, vol. 40, no. 5-6, pp. 499-508, 2004.

[5] A.-M. Wazwaz, "The sine-cosine method for obtaining solutions with compact and noncompact structures," Applied Mathematics and Computation, vol. 159, no. 2, pp. 559-576, 2004.

[6] D. M. . Mothibi and C. M. . Khalique, "On the exact solutions of a modified Kortweg de Vries type equation and higher-order modified Boussinesq equation with damping term," Advances in Difference Equations, vol. 2013, article 166, 2013.

[7] Z. Dai, J. Huang, M. Jiang, and S. Wang, "Homoclinic orbits and periodic solitons for Boussinesq equation with even constraint," Chaos, Solitons \& Fractals, vol. 26, no. 4, pp. 1189-1194, 2005.

[8] H. Jafari, A. Borhanifar, and S. A. Karimi, "New solitary wave solutions for the bad Boussinesq and good Boussinesq equations," Numerical Methods for Partial Differential Equations, vol. 25, no. 5, pp. 1231-1237, 2009.

[9] M. Rafei, D. D. Ganji, H. R. Mohammadi Daniali, and H. Pashaei, "Application of homotopy perturbation method to the RLW and generalized modified Boussinesq equations," Physics Letters A, vol. 364, no. 1, pp. 1-6, 2007.

[10] T. A. Abassy, M. A. El-Tawil, and H. El-Zoheiry, "Modified variational iteration method for Boussinesq equation," Computers \& Mathematics with Applications, vol. 54, no. 7-8, pp. 955-965, 2007.

[11] M. Javidi and Y. Jalilian, "Exact solitary wave solution of Boussinesq equation by VIM," Chaos, Solitons and Fractals, vol. 36, no. 5, pp. 1256-1260, 2008. 
[12] N. A. Kudryashov, "Simplest equation method to look for exact solutions of nonlinear differential equations," Chaos, Solitons and Fractals, vol. 24, no. 5, pp. 1217-1231, 2005.

[13] H. Jafari, N. Kadkhoda, and C. M. Khalique, "Travelling wave solutions of nonlinear evolution equations using the simplest equation method," Computers \& Mathematics with Applications, vol. 64, no. 6, pp. 2084-2088, 2012.

[14] L. A. Ostrovsky, "Nonlinear internal waves in a rotating ocean," Okeanologiya, vol. 18, pp. 181-191, 1978 (Russian), Oceanology vol. 18, 119-125, 1978.

[15] M. Duranda and D. Langevin, "Physicochemical approach to the theory of foam drainage," European Physical Journal E, vol. 7, pp. 35-44, 2002.

[16] G. W. Bluman and S. Kumei, Symmetries and Differential Equations, vol. 81, Springer, New York, NY, USA, 1989.

[17] P. J. Olver, Applications of Lie Groups to Differential Equations, vol. 107, Springer, New York, NY, USA, 2nd edition, 1993. 


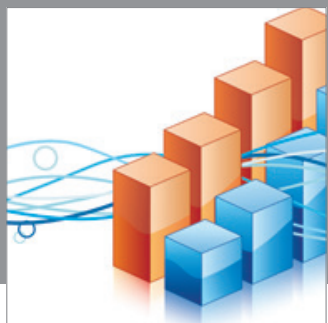

Advances in

Operations Research

mansans

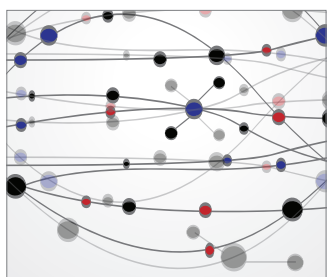

The Scientific World Journal
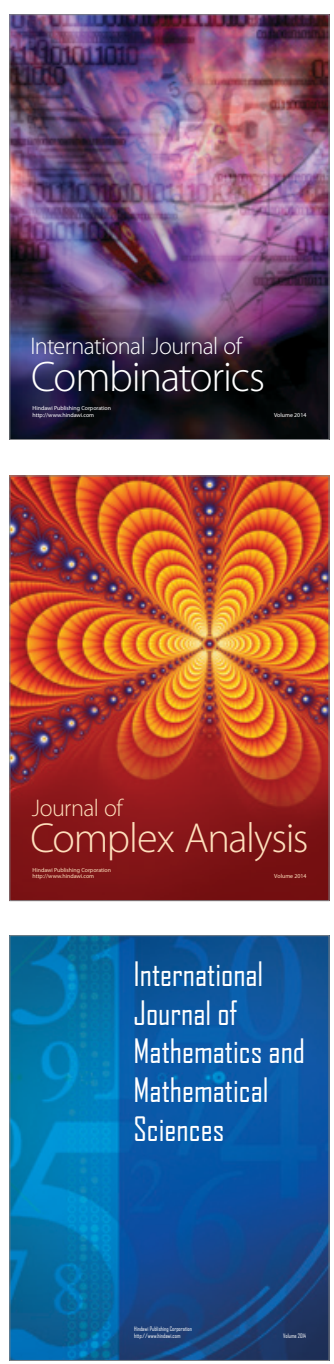
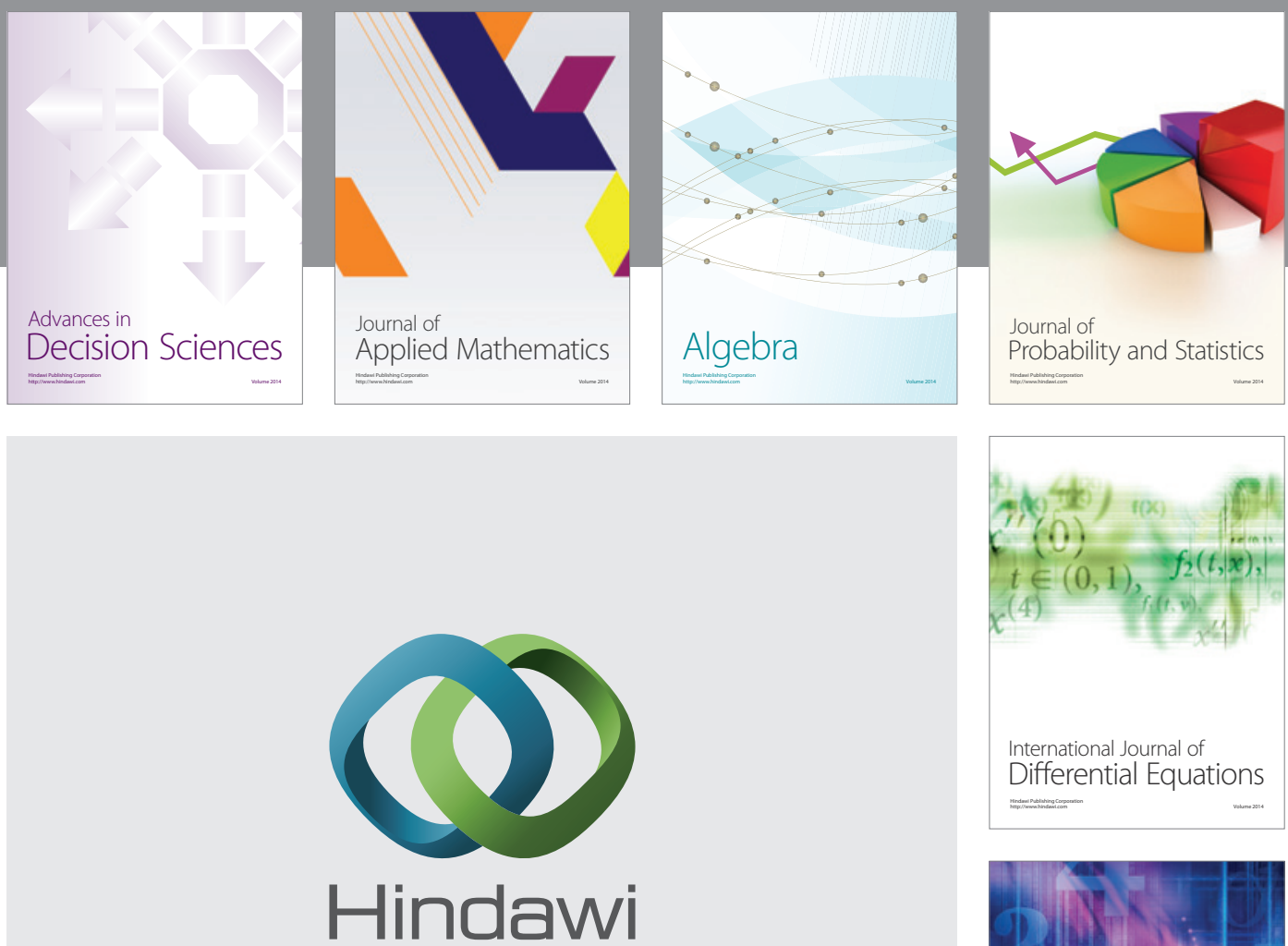

Submit your manuscripts at http://www.hindawi.com
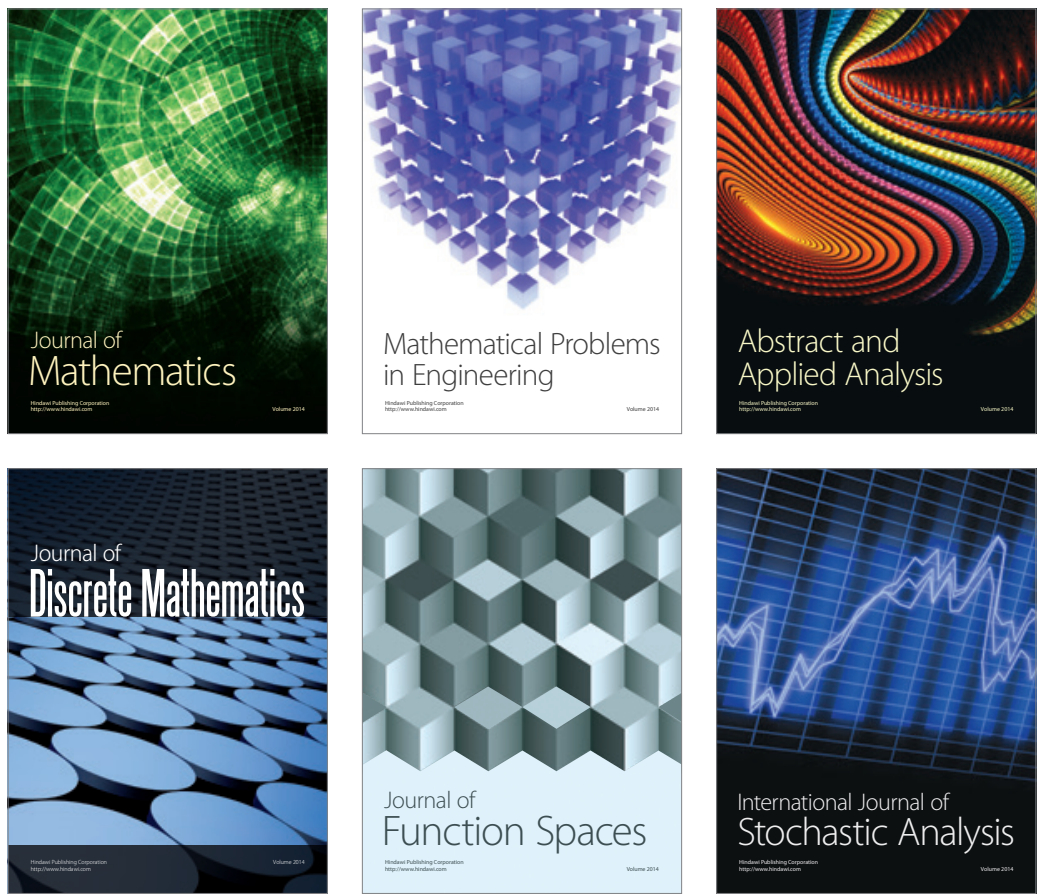

Journal of

Function Spaces

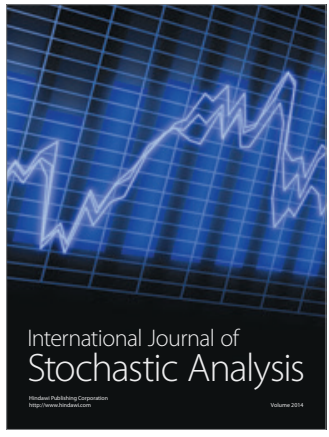

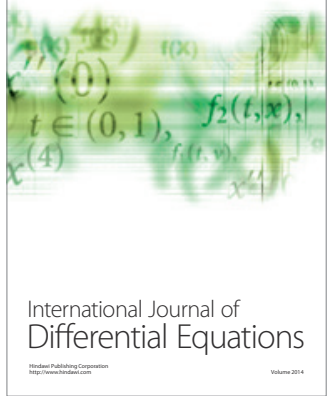
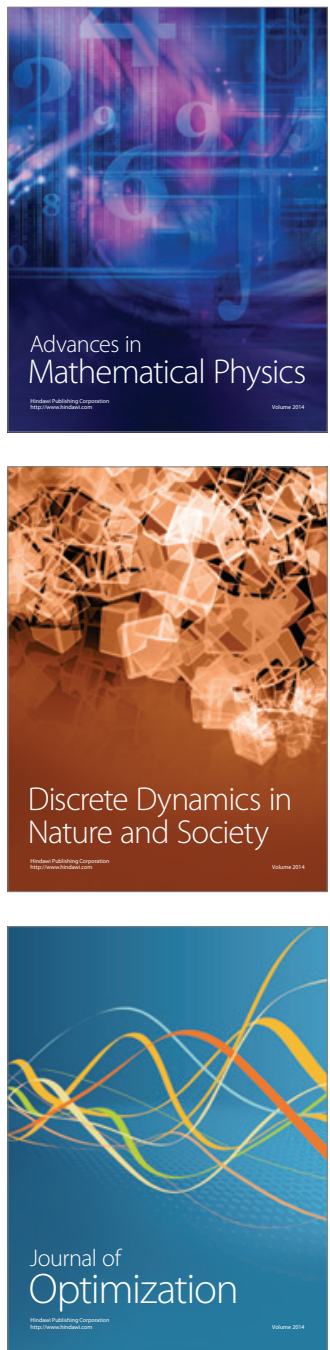